\title{
Automated Low Frequency Load Cutoff
}

P. Top, I. Hussain, M. Clower, R. Barnard, N. Luong

June 12, 2013

North American Power Symposium

Manhattan, KS, United States

September 22, 2013 through September 24, 2013 
This document was prepared as an account of work sponsored by an agency of the United States government. Neither the United States government nor Lawrence Livermore National Security, LLC, nor any of their employees makes any warranty, expressed or implied, or assumes any legal liability or responsibility for the accuracy, completeness, or usefulness of any information, apparatus, product, or process disclosed, or represents that its use would not infringe privately owned rights. Reference herein to any specific commercial product, process, or service by trade name, trademark, manufacturer, or otherwise does not necessarily constitute or imply its endorsement, recommendation, or favoring by the United States government or Lawrence Livermore National Security, LLC. The views and opinions of authors expressed herein do not necessarily state or reflect those of the United States government or Lawrence Livermore National Security, LLC, and shall not be used for advertising or product endorsement purposes. 


\title{
Automated Low Frequency Load Cutoff
}

\author{
Philip Top \\ Lawrence Livermore National Lab \\ Livermore, CA, \\ Israr Hussain, Matthew Clower, Robert Barnard and Norman Luong \\ Univeristy of California Merced \\ Merced, CA \\ Email:phlptp@ieee.org
}

\begin{abstract}
The power grid is changing, new types of generation are increasingly coming on line at both the commercial and consumer scale. As technology improves, energy harvesting, by various means, will force the grid to adapt. Traditional means of control and management may become strained or inadequate for the future grid. The grid is also becoming smarter with increasing interaction between utilities and consumers, and as it becomes more interconnected and interactive, new threats from these interconnections arise. The drive to reduce costs and make the grid more efficient will inevitably push the operation of the grid closer to the safe limits making it increasingly likely small triggers will cause wide scale problems. One potential solution is making the system controls layered with a series of different control mechanisms including both automated and centralized systems. This article explores and demonstrates the development and potential impact of one such consumer level control mechanism. Specifically, we developed a prototype system by which non-essential, non-time critical "dumb" loads can be made to respond to detected events observable in the system frequency, and thereby assist in the recovery from larger events. This system responds in a totally automated fashion without communications or control signals, and
\end{abstract}

This document was prepared as an account of work sponsored by an agency of the United States government. Neither the United States government nor Lawrence Livermore National Security, LLC, nor any of their employees makes any warranty, expressed or implied, or assumes any legal liability or responsibility for the accuracy, completeness, or usefulness of any information, apparatus, product, or process disclosed, or represents that its use would not infringe privately owned rights. Reference herein to any specific commercial product, process, or service by trade name, trademark, manufacturer, or otherwise does not necessarily constitute or imply its endorsement, recommendation, or favoring by the United States government or Lawrence Livermore National Security, LLC. The views and opinions of authors expressed herein do not necessarily state or reflect those of the United States government or Lawrence Livermore National Security, LLC, and shall not be used for advertising or product endorsement purposes.

Lawrence Livermore National Laboratory is operated by Lawrence Livermore National Security, LLC, for the U.S. Department of Energy, National Nuclear Security Administration under Contract DEAC52-07NA27344

LLNL-CONF-638804 thus provide a measure of immunity from communications problems and could replace a small amount of reserves that would otherwise be required. We examine here the construction, operation and potential benefits of such a device.

\section{INTRODUCTION}

Power grids are a very complex system of interconnected components, comprising generators, consumers, and a transmission and distribution system to connect everyone together. Traditionally, power generation has primarily consisted of large spinning generators that convert kinetic or potential energy from steam or water into electrical energy which can be transmitted via conductors. In recent years, direct conversion technology such as wind and photovoltaic has become more mainstream and cost effective particularly with government incentives. These new technologies represent a fundamentally different way of powering the grid. They connect to the power grid through silicon instead of through a giant spinning mass of conductors. Which can reduce the cost and expense of connecting generation on the grid, but also presents some new challenges to the operation and control of the power grid. The spinning motion of generators acts as a brake on the system and naturally opposed any changed in power level adding stability. Automatic control system on the generators acted to correct mismatches between power and load.

With new technologies displacing these spinning generators, the inertia of the system will decrease along with the associated control systems that acted to correct the deviations. The marker of these deviations is observed in the small variations in frequency of the grid. These deviations can be observed anywhere that is connected to the grid with simple, readily available equipment [1]. The next step after observing is acting on that information. The frequency information from the power grid acts as a real-time communication system broadcasting the state of the power grid on an analog channel to every connected 
outlet. This signal can then be used as an automatic control input.

Some such controllers are already in existence. Devices can be built cheaply enough that they could be incorporated into appliances as a trigger for very short-term demand response systems. Pacific Northwest National Labs has developed some components that could be added to appliances to allow them to respond rapidly to events on the grid by detecting the voltage or frequency [2]. But this sort of device, while widely applicable to new appliances cannot be applied to existing devices, or simple devices with no computer interface. Other schemes have made use of the capabilities of new smart meters to dynamically control various appliances [3], this again requires additional components to be installed with the capability of communicating with a smart meter.

There is a fraction of appliances and loads that are generally left on all the time because they occasionally need power to maintain a relatively constant temperature, level, or other slowly varying physical property. Some examples are heaters, pumps or chillers, which could be turned off quickly if there was a mechanism in place to do, and would suffer no ill effects upon restarting. Conceptually at least these loads could be used as part of a control mechanism for the grid to assist in recovery from larger events.

\section{DEVICE DESIGN AND CONSTRUCTION}

To demonstrate this concept a prototype device was designed and built to turn off the power to an outlet, based on signals from that outlet. The goal of the team was to design a device capable of monitoring the frequency of a wall outlet and selectively switching power. A schematic of the final design is shown Figure 1. There are four main components to the control circuit. There is a power supply, which brings in a $12 \mathrm{~V}$ AC signal from the transformer, runs it through a bridge rectifier, and utilizes low pass filters and a LM7805 voltage regulator to output a steady $5 \mathrm{~V}$ DC signal for powering most of the circuit. An opto-isolator acts as an analog to digital converter by translating the sine-wave AC signal into a $5 \mathrm{~V}$ step signal. The microcontroller then processes this step signal and controls the relay accordingly. This system was designed in discrete sections to allow for modular testing and analysis. Each subsystem was tested and verified independently before the entire system came together, and each subsystem can be checked independently if any part of the system fails.
The bridge rectifier output is fed into a ripple filter and a $7805 \mathrm{~s} 5 \mathrm{~V}$ regulator to power the microcontroller. The output is filtered to maintain a constant $5 \mathrm{~V}$ signal with low noise using the regulator, a $100 \mu \mathrm{F}$ electrolytic capacitor, and a $220 \mathrm{nF}$ ceramic capacitor for high frequency rejection.

The frequency is measured using zero crossings of the $60 \mathrm{~Hz}$ power signal. The output from the transformer is fed into an opto-isolator. The opto-isoltor acts to separate the mains signal from the circuitry and convert the $60 \mathrm{~Hz}$ signal into a square wave. The opto-isolator output is passed into an amplifier circuit which fixes it at 0 or $5 \mathrm{~V}$ for input to the microcontroller, a signal diagram of this process is shown in Figure 2.

The controller logic was designed with the Arduino development environment to measure the time period between two subsequent rising or falling edges. The logic was later transferred to the smaller ATtiny to reduce board footprint and reduce power consumption. When the microcontroller detects a low frequency measurement it triggers a relay through the use of FET switch to drive the relay. The relay behaves in a similar fashion to ground fault circuit interrupt(GFCI) outlets commonly in use in kitchens and bathrooms.

The system, as built, consumes approximately $200 \mathrm{~mW}$ of power while in monitoring mode and $900 \mathrm{~mW}$ when in active state to control the switching relay. With further design revisions the power consumption can likely be brought even lower by using a $3 \mathrm{~V}$ microprocessor and other lower power components.

To test the system the components were placed on a PC board and the system was tested for a period of time to examine the susceptibility to false alarms. Though the test period was limited there was no observed false alarms during the period of the test.

\section{SIGNAL PROCESSING}

The frequency of the power system is computed by measuring the time from one rising edge to the next rising edge. These measurements are averaged over a second to reject noise and keep the system from reacting to spurious signals. Clock error in the microcontroller and clock drift are accounted for computing a slow moving average of the signal. Since the device is intended to respond to sudden, large changes in the frequency, detecting changes in the frequency from a long average functions nearly as well as having an absolute clock. In the United States, at least, the grid frequency is 


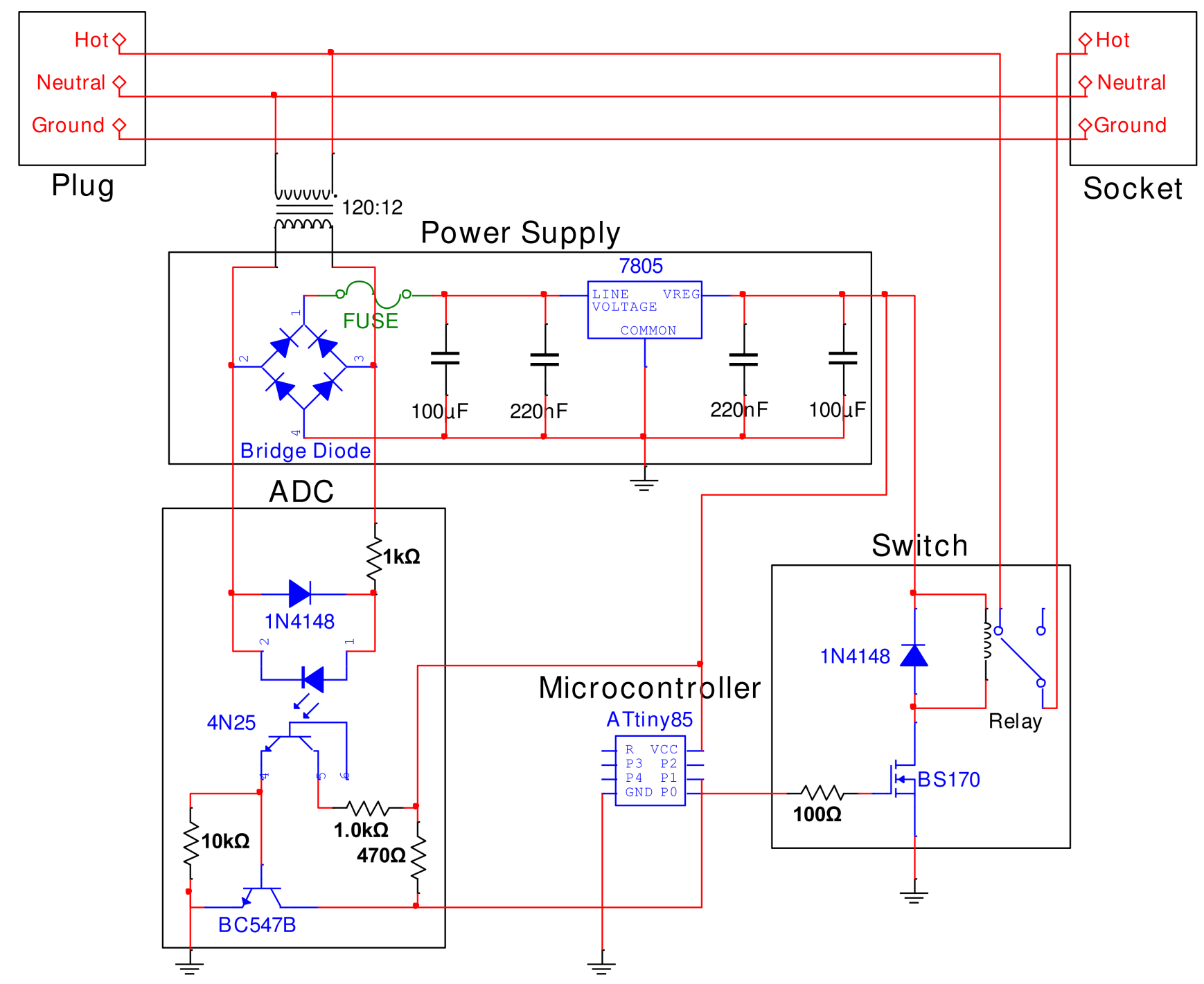

Fig. 1. Control circuit schematic

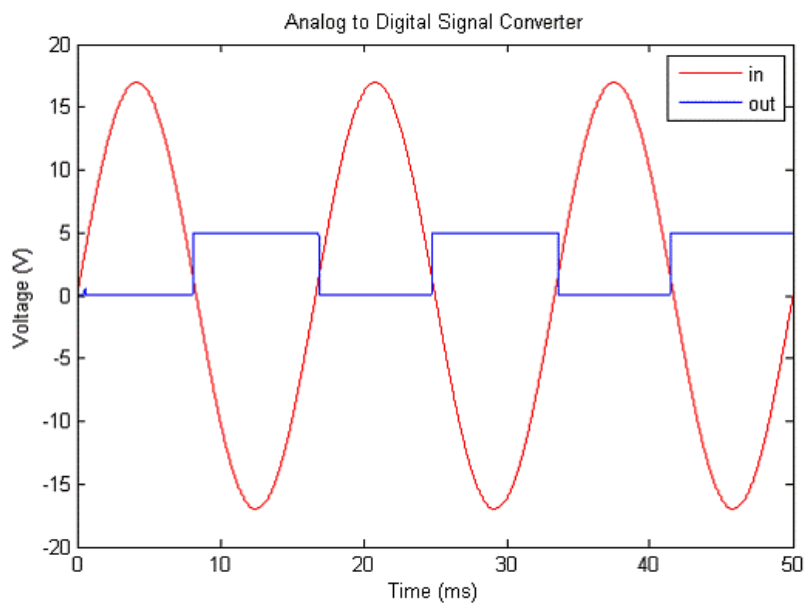

Fig. 2. Zero Crossing Example generally very close to $60 \mathrm{~Hz}$ over long periods. This stability provides some measure of immunity to clock and temperature drift in the microcontroller. The algorithms as specified were generally able to detect variations in frequency as low as $0.001 \mathrm{~Hz}$ and were reliably able to estimate the frequency to within $0.01 \mathrm{~Hz}$, based on tests using a calibrated signal generator.

The conditions used to trigger an event can be adjusted in the controller. These parameters include the trigger frequency, minimum out of bounds time, outage duration, and variability in recovery time. Once a frequency measurement falls below the trigger threshold for the specified duration the switch is toggled and power is cut to the load. While the switch is in the off position the system continually monitors the frequency and once 


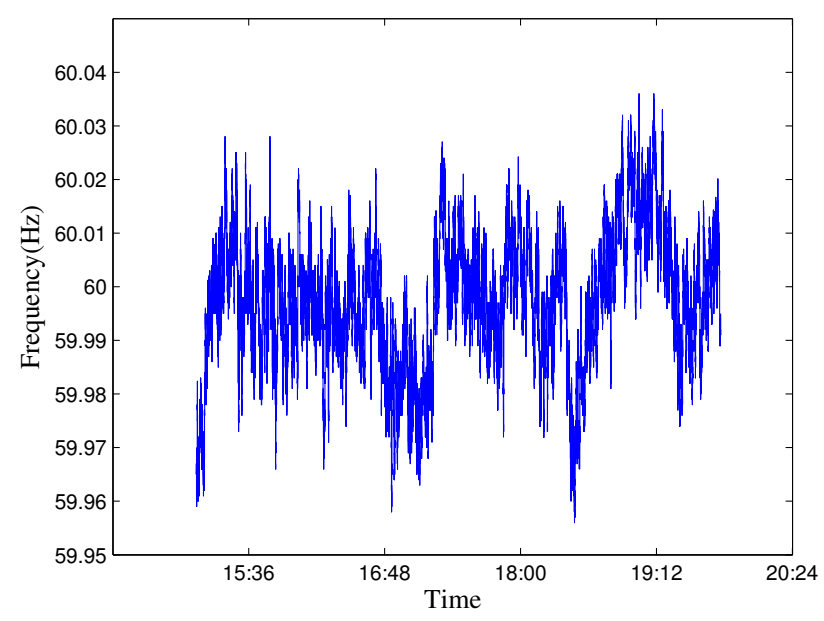

Fig. 3. Example of typical frequency variations

the outage time has elapsed and the frequency returned to nominal values, a random timer is started before turning the switch back on. This random timer prevents all devices from turning back on simultaneously and potentially creating other problems from sudden power inreases.

\section{A. Grid Frequency Variations}

In the United States the frequency of the grid is controlled to a very tight tolerance. The magnitude of the variations is roughly correlated with the amount of load on the grid and how well the generators respond to controls and schedules. An example of a typical frequency variation over a couple hours is shown in Figure 3. The trigger frequency of the switch must be set at a point where it is triggered only on rare occasions, but often enough that it can make a legitimate difference in times of grid stress and be economically viable by alleviating some of the need for system reserves.

Since such events are random, the conditions must be thought of in terms of probability. Looking at the record of grid frequency in the western United States we can determine the probability of the frequency falling below a certain level. The daily probability of an event is shown in Figure 4. This chart would indicate that the set point should be somewhere between 59.91 and $59.93 \mathrm{~Hz}$ to trigger only on larger events. This would imply the device might trigger on the order of once every 10 days for a few minutes.

Large grid events that would trigger the system response follow a similar pattern. One example of a large

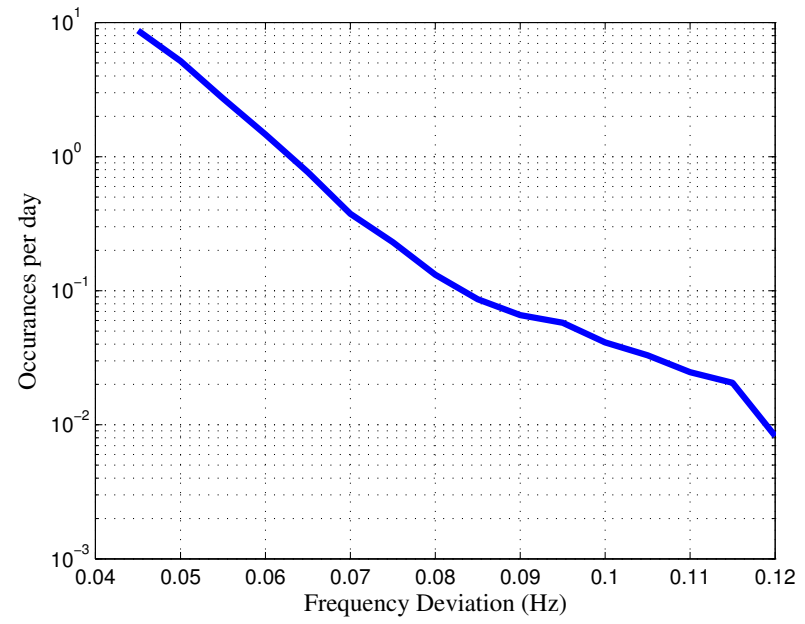

Fig. 4. Probability of Frequency Deviations

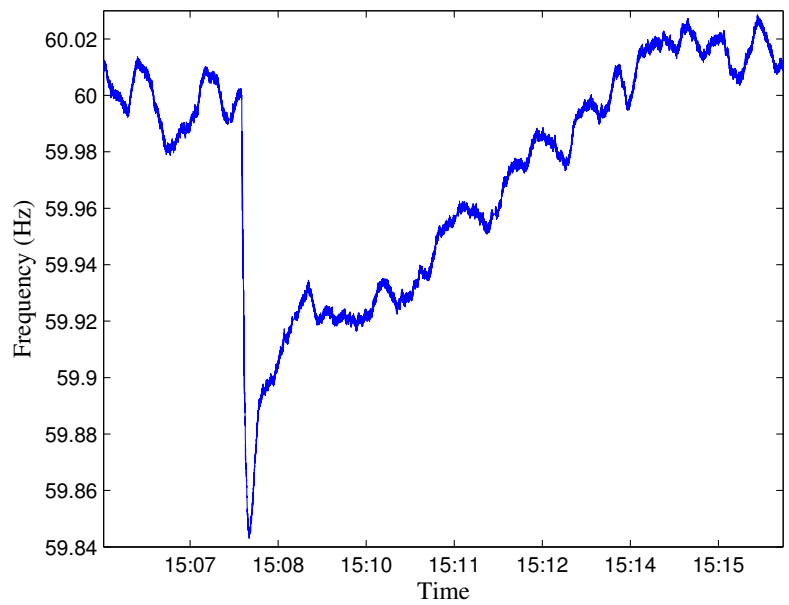

Fig. 5. Grid event on April 01, 2013

event is shown in Figure 5. Immediately following this event, the frequency falls very rapidly. The fall is arrested at the nadir frequency by the governors on the generators stabilizing the frequency at a new level. Over the next few minutes the reserve generators kick in to restore system frequency to nominal levels. A fast acting load cutoff could in theory reduce the magnitude of the drop in frequency, and thereby give the primary control actions of the system more time to react, reducing the probability of a cascading event or allowing slower, less expensive units to act as reserves for the same system performance. For the system to be useful it would have to respond within seconds after an event, and keep the load off for up to 15 minutes while the system recovers. The 15 minute period is based on NERC criteria for a balancing 


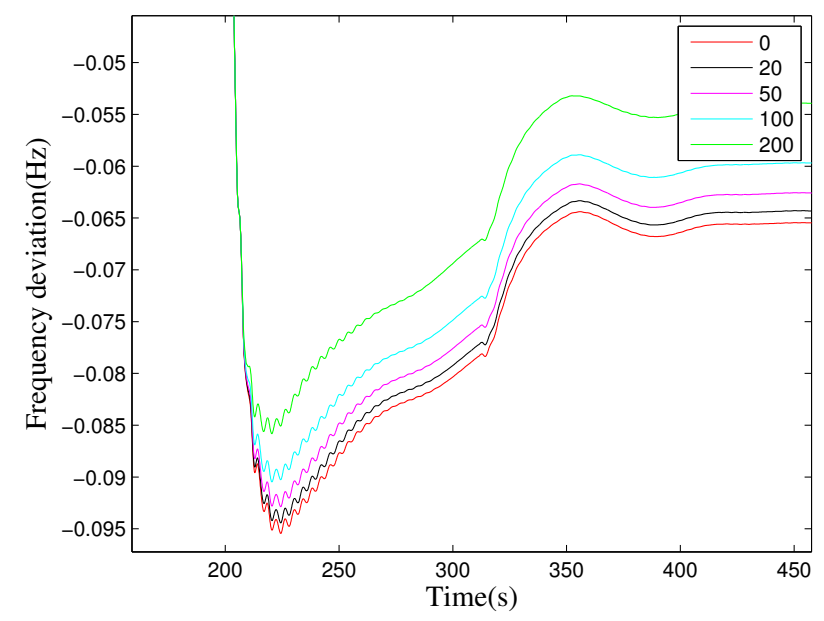

Fig. 6. Simulation results with varying levels of load cutoff

authority to recover from a system event [4].

\section{Simulations}

A simulation was set up using a reduced model of the Western Electric Coordinating Council (WECC) system projected for the year 2020 with a high renewable portfolio. The generators were aggregated into 5 areas in the WECC: California, the Desert Southwest, the Northwest, Mountains, and Canada. The generators were aggregated by type including gas, hydro, coal, nuclear, and renewables, each was given properties related to the generator type. The model was intended to provide an approximate estimate of the impact of the device. To simulate an event, a $2000 \mathrm{MW}$ load was turned on and the resulting grid frequency observed under several scenarios. First with no load cutoff, and with 20, 50, 100, and $200 \mathrm{MW}$ of load cutoff. The results of the simulation are shown in Figure 6.

The load is fast acting enough, that it has the effect of making the contingency less severe by an amount equal to the amount of load being cut off. Which is pretty much as expected. It has the effect of making the load frequency responsive and increasing the frequency response of the system, which could be increasingly valuable as power grids move from centralized generation to significant amounts of renewable energy and distributed generation. The frequency response shows that this type of load has the capability of functioning much as spinning reserves are used in the system and potentially lowering the requirement for such reserves in favor of slower acting reserves. Since this load reduction would be short lived it could not reduce the need for actual generation reserves but it could allow slower units to act as such reserves and thereby lower the cost.

\section{LOAD ANALYSIS}

The types of loads that could be triggered by such a device are primarily devices that are on all the time, and deal in some way with regulation of some kind such as temperature, flow, or level. Appliances such as hot water heaters, refrigerators, chest freezers, heaters, air conditioners, pumps, and other devices of a similar nature, may fall under this category. Many of these loads are on/off devices that are regulated with a thermostat of some kind and limited other features. Many of these units can tolerate occasional power cutoff with no ill effect.

For instance in California according to the Energy Information Archive residential energy consumption survey [5] there are approximately 1.3 million electric water heaters in the state of California. Each of them uses on average $214 \mathrm{~W}$ of power. The actual consumption pattern is periodic with occasional high load and off most of the time. With enough devices the power consumption should average out. Hypothetically, this could represent $278 \mathrm{MW}$ of power that could be triggered offline by a low frequency event if all 1.3 million electric water heaters had such devices as we propose installed between the power and water heater. In winter, electric heaters might be a candidate for use and in the summer air conditioners could be used. Other types of loads such as pool pumps or spa heaters might also be candidates.

Some of the loads described here are also candidates for other demand response system but would require much more expensive communications infrastructure and control hardware, if that infrastructure were installed the loads could in theory be used in a much more controlled fashion.

\section{ECONOMIC FEASIBILITY}

The unit as described here can be built for approximately $\$ 14$ in parts in batches of 1000 . With some additional design effort it is quite reasonable to create the product in the same form factor as a typical GFCI outlet or as a block that fits over a standard outlet and priced similarly to that of common GFCI outlets. The majority of the cost is in the transformer and the high power switch to cutoff the power. If used on water heaters, the total cost would come to around $\$ 70$ per $\mathrm{KW}$ of power reduction capacity. Installation cost and 
end-user payments could make this somewhat higher. If installed in sufficient numbers and proved effective the load reduction could shift some of the currently required spinning reserve capacity to non-spinning reserve at a cost savings. In California, in 2012, prices averaged about $\$ 4.18 / \mathrm{MW}$ for spinning reserve and \$1.49/MW for non-spinning reserve [6]. If, by the use of the frequency tripped load $20 \mathrm{MW}$ of spinning reserve can be safely shifted to non-spinning with no reduction in system reliability, then that would result in a savings of roughly $\$ 0.5$ million per year at an initial cost of around $\$ 1.0$ million if the per unit cost could be brought to around $\$ 10$. In 2011 the prices were $\$ 8.34 / \mathrm{MW}$ for spinning reserve and $\$ 1.17$ for non-spinning reserve, at these prices the annual revenue would be $\$ 1.25$ million. Prices could reasonably be expected to rise in coming years as increased renewable generation puts extra stress on the system and increases demand for units capable of fast changes. If these costs, prices, and effectiveness established here proved legitimate, it might even be enough to justify a small annual credit or payment to consumers willing to install the device.

\section{CONCLUSION}

The power grid is changing, the production of energy is becoming more varied and traditional means of control may not function as well in the past. Consumer interaction with the electric infrastructure will also increase from variable pricing schemes and distributed energy generation. It might, therefore, make sense to make consumers part of the solution. Especially, given the negligible impact of brief occasional power cuts to many appliances.

Devices such as described here may also play a role in microgrids or other smaller grids. The frequency cutoff would need to be shifted to match the characteristics of the power grid, but having frequency responsive load that responds quickly to changes in frequency could provide an additional means of stabilization on smaller grids.

As the mix of generation changes and becomes more diverse, there is a need for additional means of control on the grid and a mixture of distributed and centralized control mechanisms. What we propose and explore here is a very inexpensive means by which some "dumb" loads on the power grid can be made function in coordination with a smart grid. And does so in a way that doesn't require communications infrastructure and virtually eliminates the risk of a cybersecurity failure due to the simplicity and independent nature of the device.

\section{ACKNOWLEDGMENT}

The authors would like to thank UC Merced for supporting this design and research project.

\section{REFERENCES}

[1] P. Top, M.R. Bell, E. Coyle, and O. Wasynczuk, "Observing the power grid: Working toward a more intelligent, efficient, and reliable smart grid with increasing user visibility," Signal Processing Magazine, IEEE, vol. 29, no. 5, pp. 24-32, 2012.

[2] N. Lu and T. Nguyen, "Grid friendly appliances - load-side solution for congestion management," in Transmission and Distribution Conference and Exhibition, 2005/2006 IEEE PES, may 2006, pp. $1269-1273$.

[3] K. Samarakoon and J. Ekanayake, "Demand side primary frequency response support through smart meter control," in Universities Power Engineering Conference (UPEC), 2009 Proceedings of the 44th International, 2009, pp. 1-5.

[4] NERC Resources Subcommittee, "Balancing and frequency control," Tech. Rep., North American Electric Reliability Corporation, Jan. 2011.

[5] U.S. Energy Information Administration, "Residential energy consumption survey,"

http://www.eia.gov/comsumption/residential/data/2009/index.html, 2013.

[6] California Indpendent System Operator, "2012 annual report on market issues and performance," Tech. Rep., California Independent System Operator, Apr. 2013. 